\title{
Preferential expression of one $\beta$-tubulin gene during flagellate development in Physarum
}

\author{
Eileen C. A. Paul, † Gary L. Buchschacher, JR, David B. Cunningham, William F. Dove \\ and TIMOTHY G. BURLAND*
}

McArdle Laboratory for Cancer Research, University of Wisconsin, 1400 University Avenue, Madison, WI 53706, USA

(Received 9 July 1991; revised 17 September 1991; accepted 19 September 1991)

\begin{abstract}
The microbial eukaryote Physarum polycephalum displays several distinct cell types in its life cycle, including amoebae, flagellates and plasmodia. Despite its relative simplicity, Physarum has a tubulin gene family of complexity comparable to that of Drosophila. We have identified $\beta$-tubulin cDNAs from Physarum that are derived from the bet $A \beta$-tubulin locus and encode $\beta 1 A$ tubulin. We have also identified a partial cDNA for the unlinked bet $B \beta$-tubulin gene, which encodes $\beta 1 B$ tubulin. The polypeptide sequences encoded by bet $A$ and bet $B$ show $99 \%$ identity, but the nucleotide sequences show only $85 \%$ identity, consistent with an ancient duplication of these genes. The bet $B$ gene is expressed in amoebae, flagellates and plasmodia, whereas bet $A$ is expressed only in amoebae and flagellates. During the amoeba-flagellate transition the level of bet $A$ transcript increases over 100fold, while the level of bet $B$ transcript changes very little. Thus Physarum has a mechanism for regulating the level of discrete $\beta$-tubulin transcripts differentially during flagellate development. A need for this differential regulation could account for the maintenance of the virtually isocoding bet $A$ and bet $B \beta$-tubulin genes.
\end{abstract}

\section{Introduction}

Most eukaryotes have multiple genes for both $\alpha$ - and $\beta$ tubulins, the principal components of microtubules (MTs). Two hypotheses have been proposed to explain the multiplicity of tubulin genes. One suggests that different tubulin genes encode polypeptides with different functional properties; these in turn would compose MTs with distinct functions (Fulton \& Simpson, 1976). The other hypothesis states that multiple tubulin genes fulfil a eukaryote's need for differential regulation, especially in different cell types (Raff, 1984). Neither of these hypotheses excludes the other.

The myxomycete Physarum polycephalum, like most eukaryotes, has several functionally distinct sets of MTs.

* Author for correspondence. Tel. (608) 262 0982; fax (608) 262 2824; email TIM@WISCPSL.BITNET.

† Present address: Department of Physiology, 719 Veterinary Research Tower, Cornell University, Ithaca, NY 14853-6401, USA.

Abbreviations: MT, microtubule.

The nucleotide sequence of cDNA $\beta 602$ has been submitted to GenBank and has been assigned the accession number M58521.
In Physarum, the distinct sets of MTs are differentially utilized in different cell types. The uninucleate amoeba utilizes microtubules in the cytoskeleton, centrioles and the open mitotic spindle. During the development of the amoeba into the flagellate, the cytoplasmic MTs change their central stellate arrangement to form a cone radiating from the anterior of the cell. The centrioles migrate to this region of the cell, where they form the basal bodies, nucleating two flagellar axonemes. The multinucleate, syncytial plasmodium, by contrast, utilizes MTs only in its intranuclear mitotic apparatus (Burland et al, , 1983).

Physarum has at least five $\alpha$-tubulin (alt) genes and three $\beta$-tubulin (bet) genes (Schedl et al., 1984b). Thus, this relatively simple eukaryote has a tubulin gene family comparable in complexity to that of Drosophila (Sanchez et al., 1980). We are studying the $\beta$-tubulin gene family of Physarum to try to understand how multiple tubulin genes may be of benefit to a simple microbial eukaryote. We show here that the multiple genes for $\beta$-tubulin show different patterns of expression in different cell types. Even two virtually isocoding $\beta$-tubulins are expressed differentially during flagellate development, consistent with the idea that multiple tubulins provide a mechanism for differential regulation. 


\section{Methods}

Cells and culture conditions. Amoebae of $P$. polycephalum strain LU352 (Dee et al., 1989) were grown in $50 \mathrm{ml}$ SDM (Cooke \& Dee, 1975 ) in $500 \mathrm{ml}$ flasks at $26^{\circ} \mathrm{C}$ in a rotary shaker at 50 r.p.m. To induce amoebae to form flagellates efficiently from axenic medium, we found it essential to use Oxoid bacteriological peptone in SDM as originally described (Cooke \& Dee, 1975), rather than Difco Bacto Soytone, which is commonly used instead. Strains CLd (Dee et al., 1989) and MA275 (Schedl et al., 1984b) have been described previously.

Induction of flagellate development. To induce flagellate development, LU352 amoebae at 4-10 $\times 10^{6} \mathrm{cells} \mathrm{ml}^{-1}$ were centrifuged at $200 \mathrm{~g}$ for $2.5 \mathrm{~min}$. Growth medium was decanted and cells were resuspended in 1 vol. SDM diluted fourfold in water; this point was taken as time zero. The culture was then shaken at 50 r.p.m., $26^{\circ} \mathrm{C}$ for $30 \mathrm{~min}$, then centrifuged at $50 \mathrm{~g}$ for $2.5 \mathrm{~min}$ and the supernatant decanted. Cells were resuspended in one volume of SDM diluted 16-fold in water and shaken at 50 r.p.m., $26^{\circ} \mathrm{C}$. Appearance of flagellates was monitored by phase-contrast microscopy of cells that were fixed by placing them on top of a layer of $1.0 \%(\mathrm{w} / \mathrm{v})$ agarose, $3.7 \%(\mathrm{v} / \mathrm{v})$ formaldehyde on a microscope slide.

Construction and screening of $c D N A$ libraries. Total RNA was isolated from cells after lysis in guanidinium isothiocyanate (Schedl $e t$ al., 1984a). Poly(A)+ RNA was prepared by oligo(dT) chromatography (Aviv \& Leder, 1972).

Two cDNA libraries, designated ML5 and ML6, were prepared. ML5 was prepared from total RNA extracted from amoebae. ML6 was prepared from poly(A)+ RNA extracted from a culture early in flagellate development, and was expected to include cDNAs of transcripts from both flagellates and amoebae. cDNA was synthesized using standard procedures for ML5 (Huynh et al., 1984) or, for ML6, using a kit (Amersham, cat. no. RPN.1257). cDNA was treated with EcoRI methylase. EcoRI linkers were then added, and after digestion with $E c o$ RI, the cDNA was size-fractionated using a $1.0 \%(w / v)$ lowmelting temperature agarose gel (for ML5), or on a BioGel A50 column (Huynh et al., 1984) (for ML6). In ML5, cDNA of 0.5-3 kb was ligated into the vector $\lambda$ gt 10 digested with EcoRI. In ML6, cDNA greater than $1 \mathrm{~kb}$ was ligated into the same vector. The phage DNA was packaged in vitro using extracts prepared by the method of Zehnbauer \& Blattner (1982) using modifications of V. Burland (University of Wisconsin, Madison, personal communication). A total of $4 \times 10^{5}$ phage plaques were obtained for ML5 and $1.4 \times 10^{6}$ for ML6. To screen the library, nitrocellulose filter replicas of phage plaques were probed with nicktranslated $\beta$-tubulin cDNAs (Rigby et al., 1977). ML5 filters were probed with a mixture of Physarum betC $\mathrm{cDNA} \beta 102$ (Burland et al., 1988) and chicken probe pT2 (Valenzuela et al., 1979). ML6 filters were probed with Physarum betC cDNA $\beta 102$ (Burland et al., 1988) and bet $B$ cDNA $\beta 502$ (from ML5). The filters were washed in $1 \times$ SSC ( $150 \mathrm{mM}-$ $\mathrm{NaCl}, 15 \mathrm{~mm}$-sodium citrate) in $0.1 \%$ sodium dodecyl sulphate. After purifying the $\beta$-tubulin-positive recombinant plaques, DNA was extracted and the size of the cDNA insert determined by EcoRI digestion and agarose gel electrophoresis. Clones from ML5 were numbered in the 500s and from ML6 in the 600s.

Subcloning $c D N A$ and sequencing strategy. The cDNAs were subcloned from $\lambda$ into the $E c o$ RI site of bacteriophage M13mp18 and were further subcloned using the Cyclone deletion system (International Biotechnologies) by the method of Dale et al. (1985) for sequencing by dideoxynucleotide chain termination (Sanger et al., 1977), using Sequenase (USB).

DNA sequences were entered into computer files manually and manipulated using DNASTAR software. DNA sequences were translated with the program TRANSLATE and protein sequence comparisons were made pairwise with the program AALIGN, which uses the Lipman method to identify homologies and introduces gaps and makes the final alignment by the protocol of Needleman \& Wunsch (1970). All percentage identities were rounded to the nearest integer.

In vitro transcription and translation. For the production of transcripts in vitro, $\beta 602$ was subcloned into the plasmid pGEM4blue (Promega), with the translation start site adjacent to the SP6 promoter. This pGEM4blue $\beta 602$ plasmid was then linearized with $X b a I$ and extracted with phenol and chloroform, precipitated with ethanol and resuspended in water. Linear pGEM4blue $\beta 602$ DNA was then transcribed in vitro using SP6 polymerase (Melton et al., 1984). About $300 \mathrm{ng}$ of transcript was translated in a wheatgerm translation system (RPN. 1 ; Amersham) in $30 \mu \mathrm{l}$ reactions containing $\left[{ }^{35}\right.$ S]methionine (SJ.204; Amersham) and 19 unlabelled amino acids. To identify the products of the transcripts, 0.06 vol. of the translation mixture was co-electrophoresed with unlabelled lysates of Physarum plasmodia and blotted onto nitrocellulose filters as described previously (Burland et al., 1988). Blots were probed with a mixture of $\alpha$-tubulin-specific monoclonal antibody YLl/2 (Kilmartin et al., 1982) and $\beta$-tubulin-specific monoclonal antibody KMX-1 (Birkett $e$ t al., 1985). Reaction with the antibodies was detected using horseradish-peroxidase-conjugated secondary antibodies. The same filters to which electrophoretically separated proteins had been blotted and immunostained were then exposed to X-ray film to detect radiolabelled translation products. Translation products were distinguished using the immunologically detected unlabelled marker tubulins from the cell lysates.

Agarose gel electrophoresis and filter hybridization. For Southern blotting, Physarum DNA from haploid strains CLd and MA275, and diploid CLd $\times$ MA275, was digested with the restriction enzyme StuI, separated by electrophoresis on a $0.7 \%$ agarose gel, partially hydrolysed by acid depurination, and transferred to nitrocellulose filters using standard procedures (Southern, 1975). Nitrocellulose filters were prehybridized, hybridized and washed under the stringent conditions described by Schedl et al. (1984b).

For Northern blotting, $3 \mu \mathrm{g}$ RNA was glyoxalated and electrophoresed on a $1.7 \%(w / v)$ agarose gel for $10 \mathrm{~h}$ at $50 \mathrm{~V}$, then transferred to a Biodyne A nylon membrane and prehybridized and hybridized as described by Schedl et al. $(1984 a)$. Membranes were washed six times for $20 \mathrm{~min}$ in $0.1 \times \mathrm{SSC}$ at $55^{\circ} \mathrm{C}$ and exposed to pre-flashed Kodak XRay film (Laskey \& Mills, 1977). RNA was quantified by scanning autoradiographs using a Biomed Instruments soft laser scanning densitometer model SL-504-XL. Linearity was assessed by densitometry of a series of different exposures of the blots.

\section{Results}

\section{Identification of $\beta$-tubulin cDNA clones}

We made two separate cDNA libraries, the first from amoebae (ML5), the second from an early stage of flagellate development (ML6), which should represent both amoebal and flagellate transcripts. All $4 \times 10^{5}$ clones from ML5 were screened; two clones hybridized to the $\beta$-tubulin probes. These were recloned to plaque purity and designated $\beta 501$ and $\beta 502$. Of $10^{5}$ clones screened from ML6, 16 hybridized to the $\beta$-tubulin probes. One clone, $\beta 602$, was recloned and used for further analysis. 
(a)

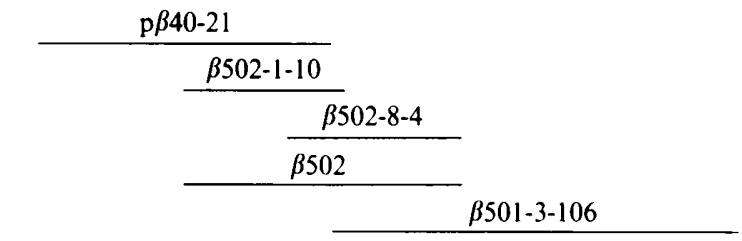

B501-33-3

$\beta 501-33-7$
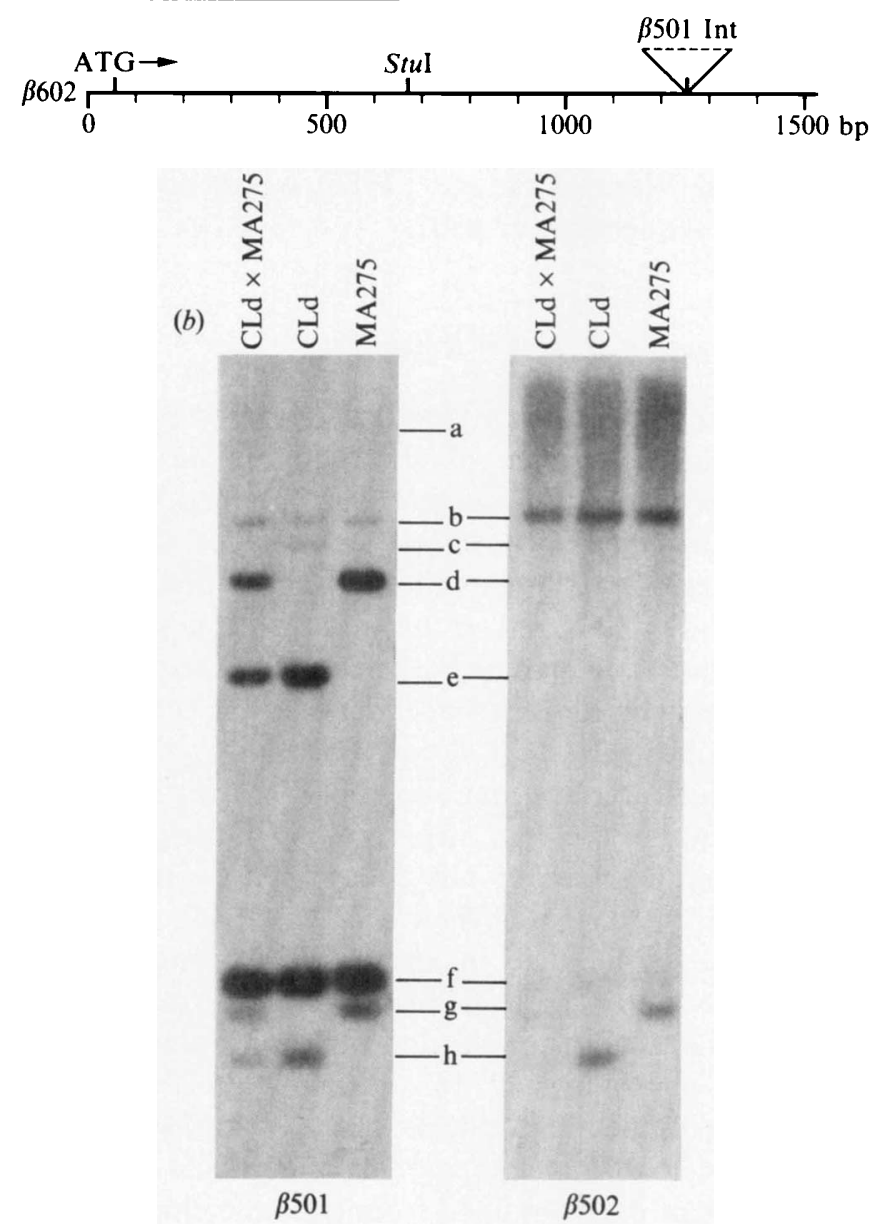

Fig. 1. Identification of the locus for the $\beta 501$ and $\beta 502$ clones. (a) cDNA probes, showing positions relative to betA cDNA $\beta 602$. $\mathrm{p} \beta 40-21$, genomic fragment of bet $B$ (Werenskiold et al., 1988); $\beta 502$, $\beta 502-1-10, \beta 502-8-4$, partial cDNA for bet $B$ and subclones; $\beta 501-3-106$, $\beta 501-33-3, \beta 501-33-7$, subclones of cDNA for bet $A$; ATG, start codon; StuI, StuI restriction site; Int, intron-like sequence. (b) Southern blots of Physarum StuI DNA digests probed with ${ }^{32} \mathrm{P}$-labelled $\beta$-tubulin cDNAs $\beta 501$ and $\beta 502$.

\section{Locus assignments}

On Southern blots of StuI-digested Physarum DNA hybridized with $\beta$-tubulin DNA probes, three pairs of bet $(\beta$-tubulin) alleles show restriction fragmint length differences between strains CLd and MA275 (Fig. 1; Schedl et al., 1984b). Bands d and e are alleles of bet $A ; \mathrm{g}$ and $\mathrm{h}$ are alleles of betB; $\mathrm{a}$ and $\mathrm{c}$ are alleles of betC
(Schedl et al., 1984b). Haploid stralns like CLd and MA275 carry only one of each of these pairs of fragments, whereas diploids like CLd $\times$ MA275 carry both (Fig. 1). There are also two monomorphic bet bands, $\mathrm{b}$ and $\mathrm{f}$, for which polymorphic restriction fragments have not been found (Schedl et al., 1984b). For locus assignments, use of DNAs from polymorphic strains helps to make locus identification unequivocal between closely migrating bands.

The $\beta 501$ cDNA probe hybridizes preferentially to alleles $\mathrm{d}$ and $\mathrm{e}$ (Fig. 1), indicating that $\beta 501$ is derived from bet $A$, and to the monomorphic band $\mathrm{f}$, indicating that band $\mathrm{f}$ may also be derived from betA. $\beta 501$ hybridizes less strongly to alleles of other loci. $\beta 502$ cDNA preferentially hybridizes to bet $B$ alleles $g$ and $h$ (Fig. 1), indicating that $\beta 502$ is derived from bet $B$, as well as to the monomorphic band $b$, indicating that band $b$ may also be derived from the bet $B$ locus. Again, $\beta 502$ hybridizes only weakly to other tubulin loci. The $\beta 602$ cDNA was assigned to the bet $A$ locus based on its sequence identity with portions of $\beta 501$ (see below).

cDNAs for betA and bet $B$ contain a Stul restriction site in codon 206 (Fig. 1), indicating that bet $A$ and bet $B$ should each yield two genomic $S t u I$ fragments in haploid strains. For bet $A$, subcloned cDNA probe $\beta 501-33-7$ (Fig. $1 a$ ), which contains only sequences $5^{\prime}$ to the StuI site, hybridizes specifically to the polymorphic bet $A$ bands $\mathrm{d}$ and $\mathrm{e}$, whereas probe $\beta 501-3-106$, containing only sequences $3^{\prime}$ to this $S t u I$ site, hybridizes specifically to the monomorphic bet $A$ band $\mathrm{f}$ (not shown). For bet $B$, the corresponding $5^{\prime}$ cDNA probe $\beta 502-1-10$ (Fig. 1) hybridizes specifically to the polymorphic bet $B$ bands $g$ and $\mathrm{h}$, while the corresponding $3^{\prime}$ probe $\beta 502-8-4$, which contains mostly sequence $3^{\prime}$ to the $S t u I$ site, hybridizes preferentially to the bet $B$ monomorphic band $\mathrm{b}$ (not shown). Thus, the polymorphic bands from bet $A$ and bet $B$ are derived from the $5^{\prime}$ ends of these genes, and the monomorphic bands are derived from the $3^{\prime}$ ends of these genes. This also confirms that the monomorphic bands are not fragments of unlinked $\beta$-tubulin genes.

\section{Gene product assignment}

To determine the product of bet $A$, RNA was transcribed from pGEM4Blue $\beta 602$ using SP6 RNA polymerase, and the resulting transcripts were translated in vitro. Unlabelled plasmodial lysates were mixed with the translation products and the mixtures were then resolved by twodimensional gel electrophoresis. Immunoblotting using the tubulin-specific monoclonal antibodies stained the $\alpha 1, \beta 1$ and $\beta 2$ tubulins present in the unlabelled plasmodial lysates (not shown). There was too little protein among the translation products to detect immunologically, but autoradiography of the immunoblot 


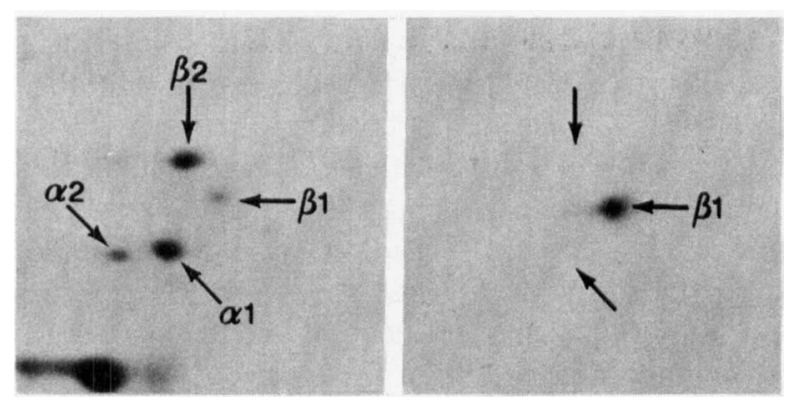

Fig. 2. Identification of the product of bet $A$. The mixture of anti- $\alpha-$ tubulin and anti- $\beta$-tubulin antibodies detected Physarum $\alpha 1, \beta 1$ and $\beta 2$ tubulins (arrows; see also Burland et al., 1988) in the mixture of cell lysates and in vitro translation products co-electrophoresed on twodimensional gels. Physarum $\alpha 2$-tubulin, detected on the left autoradiogram, does not have an epitope that $\mathrm{YLl} / 2$ recognizes (Walden $e t$ al., 1989). The blots (not shown) served to determine the precise orientation for comparison of blot and autoradiogram. The left panel shows the autoradiogram of the two-dimensional immunoblot of in vitro translation products of plasmodial poly(A) ${ }^{+}$RNA; this RNA was isolated from the $\mathrm{G} 2$ phase of the cell cycle, when tubulin mRNAs are abundant (Schedl et al., 1984a). The right panel shows the corresponding autoradiogram of the immunoblot for the in vitro translation product of cDNA $\beta 602$. Isoelectric focussing is from left (basic) to right (acidic); denaturing polyacrylamide gel electrophoresis is from top to bottom. The major radioactive translation product of $\beta 602$ is clearly $\beta 1$ tubulin. No signal in the vicinity of the other tubulins was detected after longer exposures of the autoradiogram. Other radioactive species detected were minor and of lower apparent molecular mass than tubulins; they may result from translation of prematurely terminated in vitro transcripts.

(Fig. 2) identified the position of the in vitro translation product relative to the immunologically detected marker tubulins in the gel sample mixture. The results show that $\beta 602$ encodes a $\beta 1$-tubulin polypeptide. $\beta 1$-Tubulin is composed of two gene products, $\beta 1 \mathrm{~A}-$ and $\beta 1 \mathrm{~B}-\mathrm{tubulin}$ (Burland $e t$ al., 1984). Since $\beta 602$ is derived from betA, and since bet $B$ encodes $\beta 1$ B tubulin (Burland et al., 1984), we can conclude that the $\beta 1$-tubulin encoded by bet $A$ is $\beta 1$ A-tubulin.

\section{Sequences of $\beta$-tubulin cDNAs}

The bet $A$ cDNA $\beta 501$ has approximately 1610 nucleotides (not shown), including an extensive open reading frame for $\beta$-tubulin. However, despite the presence of poly(A) $3^{\prime}$ to the coding region, $\beta 501$ contains an intronlike sequence between nucleotides 1210 and 1318 , and we experienced difficulties in accurately sequencing this region of the cDNA. We therefore do not report the sequence for this cDNA. $\beta 501-33-3$ (Fig. 1) is a subclone of $\beta 501$ which contains 730 base pairs from the $5^{\prime}$ coding region of the betA gene; this subclone hybridizes specifically to betA on Southern blots (not shown), so it was chosen as a DNA probe for Northern blotting.
cDNA $\beta 602$ has 1512 nucleotides, consisting of 53 nucleotides of $5^{\prime}$ untranslated sequence, an open reading frame of 445 codons for $\beta 1$ A-tubulin, 124 nucleotides of $3^{\prime}$ untranslated sequence and 17 nucleotides of poly(A) tail. Sequence analysis indicated that $\beta 602$ is derived from the same gene as $\beta 501$, i.e. bet $A$.

$\beta 502$ is approximately 600 nucleotides long (not shown). Comparison of preliminary nucleotide sequence for $\beta 502$ with that for bet $B$ genomic clone $\mathrm{p} \beta 40-21$ (Werenskiold et al., 1988) confirms that $\beta 502$ is derived from bet $B$ and starts at codon 103 of this gene. Since most of the bet $B$ sequence covered by $\beta 502$ is already published (Werenskiold et al., 1988), we did not complete rigorous sequencing of $\beta 502$.

\section{Comparisons of $\beta$-tubulin sequences}

Singhofer-Wowra et al. (1986) determined $90 \%$ of the amino acid sequence of $\beta 1$ tubulins purified from Physarum amoebae. Evidence was obtained for heterogeneity at only one amino acid residue (SinghoferWowra et al., 1986) in this mixture of $\beta 1 \mathrm{~A}$ and $\beta 1 \mathrm{~B}$ polypeptides (Burland et al., 1984), at position 283, where serine and alanine both occurred. The amino acid sequence deduced from the sequence of bet $A$ CDNA $\beta 602$ (Fig. 3) illustrates that serine-283 originates from this gene, which implies that alanine-283 comes from bet $B$. Translation of the $\beta 602$ sequence fills in the gaps of the $\beta 1 \mathrm{~A}$ tubulin sequence not determined by the protein sequencing (Singhofer-Wowra et al., 1986). We found four more differences in amino acids between the $\beta 1$ sequence derived from direct protein sequence of amoebal $\beta$-tubulin and the deduced amino acid sequence of $\beta 602$, at residues 39, 165, 196 and 238 (Fig 3).

In Fig. 4 , the nucleotide sequence of $\beta 602$ (bet $A$ ) from codons 4 to 204 is compared to the partial nucleotide sequence of bet $B$ obtained from genomic clone $\mathrm{p} \beta 40-21$ (Werenskiold et al., 1988). While the amino acids show $99.5 \%$ identity, the nucleotide sequence shows only $85 \%$ identity. Thus although bet $A$ and bet $B$ genes encode virtually identical proteins, there has been considerable drift in their nucleotide sequence and codon usage.

\section{Expression of bet $A$ and bet $B$}

In order to examine the expression patterns of the bet $A$ and betB genes in Physarum, RNA was isolated from amoebae, flagellates and plasmodia, and analysed by Northern blotting using the bet $A$-specific $\mathrm{CDNA}$ probe $\beta 501-33-3$ (Fig. 1) and cDNA probe $\beta 502$, which hybridizes to both bet $A$ and bet $B$ on Southern blots.

The bet $A$ gene is expressed at only a low level in amoebae but the level of the betA transcript increases 


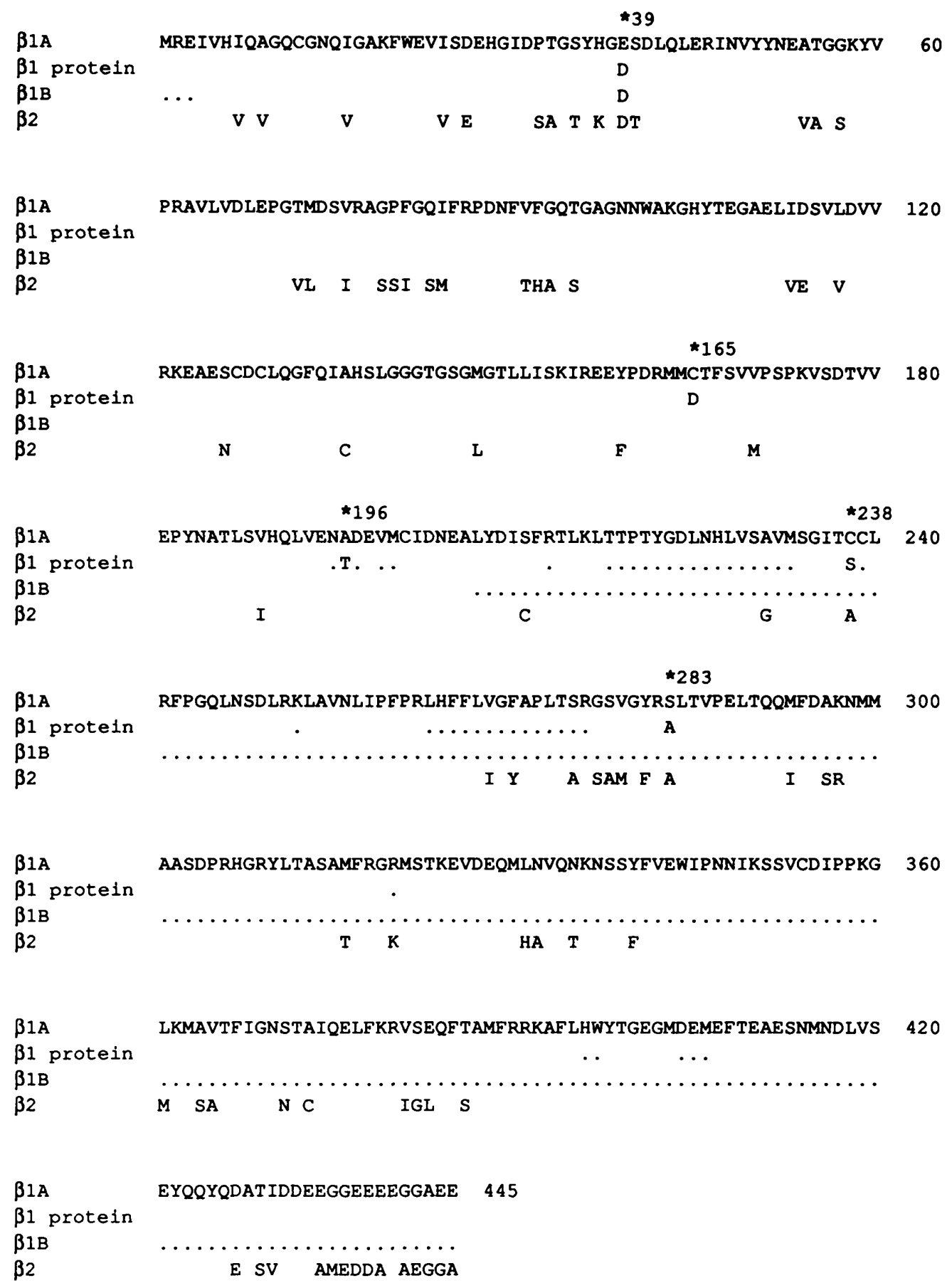

Fig. 3. Alignment of the amino acid sequences of Physarum $\beta$-tubulins. $\beta 1 \mathrm{~A}$ is the sequence deduced for the bet $A$ product from cDNA $\beta 602 ; \beta 1$ protein is the sequence derived from purified amoebal protein (Singhofer-Wowra et al., 1986); $\beta 1$ B is the sequence deduced for the bet $B$ product from partial genomic clone $\mathrm{p} \beta 40-21$ (Werenskiold $e t$ al., 1988); and $\beta 2$ is the sequence deduced for the betC product (Burland et al., 1988). All sequences were aligned with that deduced for Physarum $\beta 1$ A tubulin. Amino acid residues, numbered starting at the amino termini, are represented by the standard one-letter codes. Only residues that differ from the $\beta 1 A$ sequence are shown; dots indicate undetermined residues, and spaces indicate identical residues. Asterisks indicate positions (numbered) specifically discussed in the text.

dramatically in the flagellate (Fig. 5). No bet $A$ expression was detected in plasmodia. Since we do not have a DNA probe specific for the betB gene, it is more difficult to assess bet $B$ expression. However, bet $B$ cDNA probe $\beta 502$ appears to detect two incompletely resolved transcripts. In the amoebal and plasmodial RNAs, $\beta 502$ 


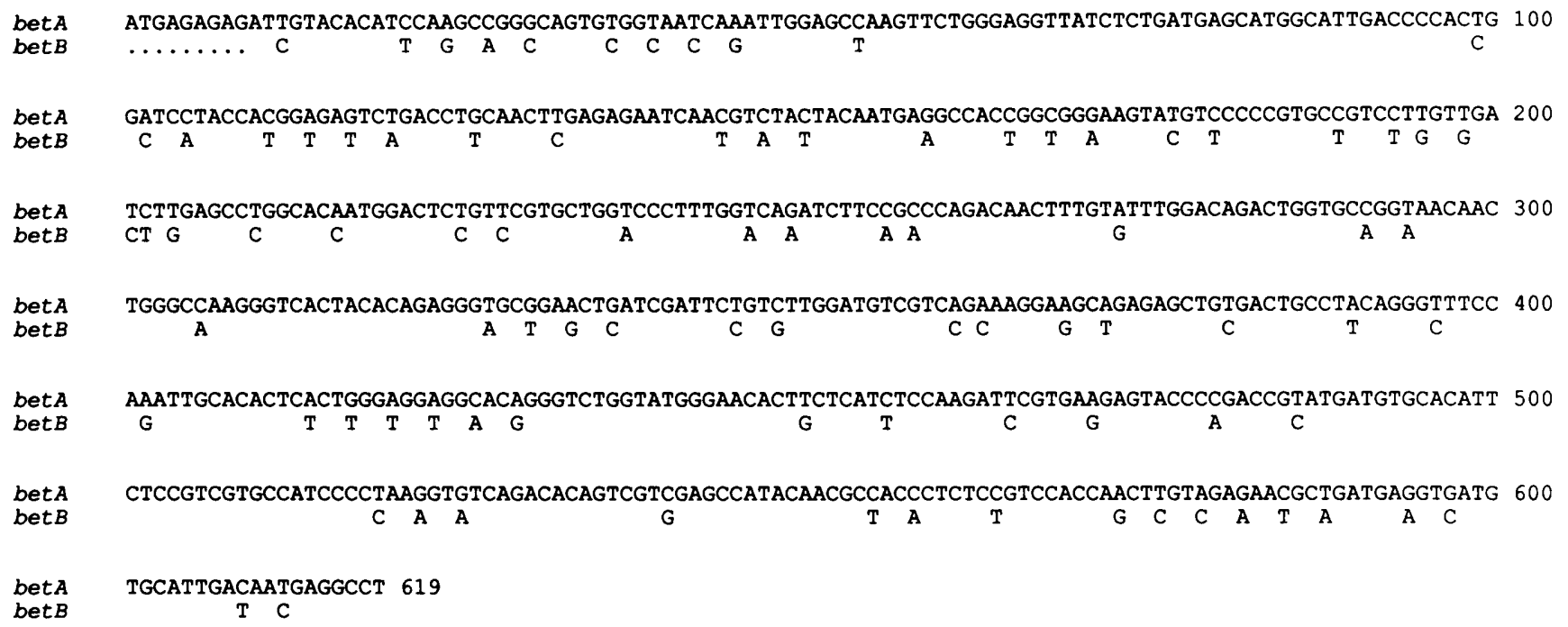

Fig. 4. Alignment of the nucleotide sequence of $\beta 602$ (bet $A$ ) with the partial genomic sequence of $\mathrm{p} \beta 40-21$ (Werenskiold et al., 1988) from bet $B$. Only residues that differ from the bet $A$ sequence are shown; dots indicate undetermined nucleotides, and spaces indicate identical nucleotides.
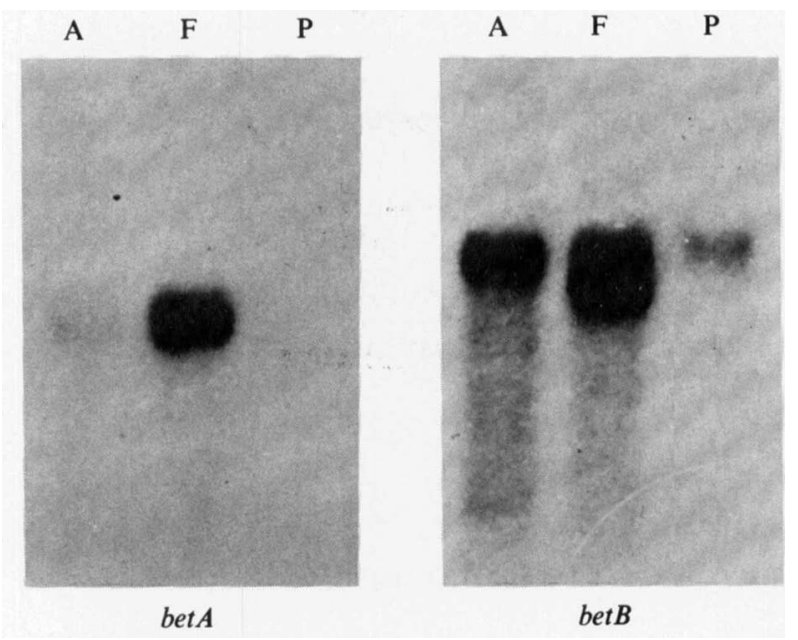

Fig. 5. Northern blot analysis of total RNA isolated from amoebae (A), flagellates (F) and plasmodia (P). The hybridization probes (Fig. 1) were $\beta 501-33-3$ for bet $A$ (left), and $\beta 502$, which detects both bet $A$ and bet $B$ (right). Exposures shown were at $-70^{\circ} \mathrm{C}$ for $18 \mathrm{~h}$. Longer exposures (not shown) reveal the low level of bet $A$ transcript in amoebae more clearly.

detects one transcript (Fig. 5), which we interpret to be from bet $B$; we already know that bet $B$ is expressed in amoebae and plasmodia (Burland et al., 1984). In flagellate RNA, $\beta 502$ detects not only the betB transcript, but also another transcript of slightly faster mobility (Fig. 5). We interpret the faster-mobility species as cross-hybridization to bet $A$ transcript, especially since this species corresponds to the position of the bet $A$ transcript detected by probe $\beta 501-33-3$ (Fig. 5, left panel). These results suggest that bet $B$ is expressed in flagellates as well as in amoebae and plasmodia.
In order to determine if the two bet transcripts detected by Northern blotting differed only in the length of poly(A) tail, the RNA from flagellates was treated with RNAase $\mathrm{H}$ in the presence of oligo(dT) to remove poly(A) tails (Mercer \& Wake, 1985). We also hoped to improve resolution of the two transcripts with this technique. However, both transcripts appeared to be shortened to the same extent (not shown), indicating that the difference between these two transcripts is not simply the length of their poly(A) tails.

\section{Kinetics of betA RNA accumulation}

We analysed how the changes in betA RNA level occurred with respect to the timing of flagellate development. Amoebae were induced to develop, and RNA was isolated at subsequent times. Northern blots of RNA were then probed with radiolabelled bet $A$-specific cDNA subclone $\beta 501-33-3$ (Fig. 6), and the levels of the bet $A$ transcripts were quantified. The peak level of bet $A$ transcript during flagellate development is 110 -fold higher than the level of bet $A$ transcript present in the amoebae. Prior to the first cytological detection of flagellates $(40 \mathrm{~min})$ the level of betA transcript was already $18 \%$ of its peak value (Fig. $6 b$ ). The peak level of bet $A$ transcript occurred when $21 \%$ of the cells were flagellates. By the time the proportion of flagellates was at a maximum ( $240 \mathrm{~min})$, the level of the bet $A$ transcript was decreasing (Fig. $6 b$ ). Control RNA detected by the cDNA probe Ppcl6, which is expressed constitutively during flagellate development (Green \& Dove, 1984), remained at approximately constant levels, while the level of actin RNA decreased slightly (not shown) when 

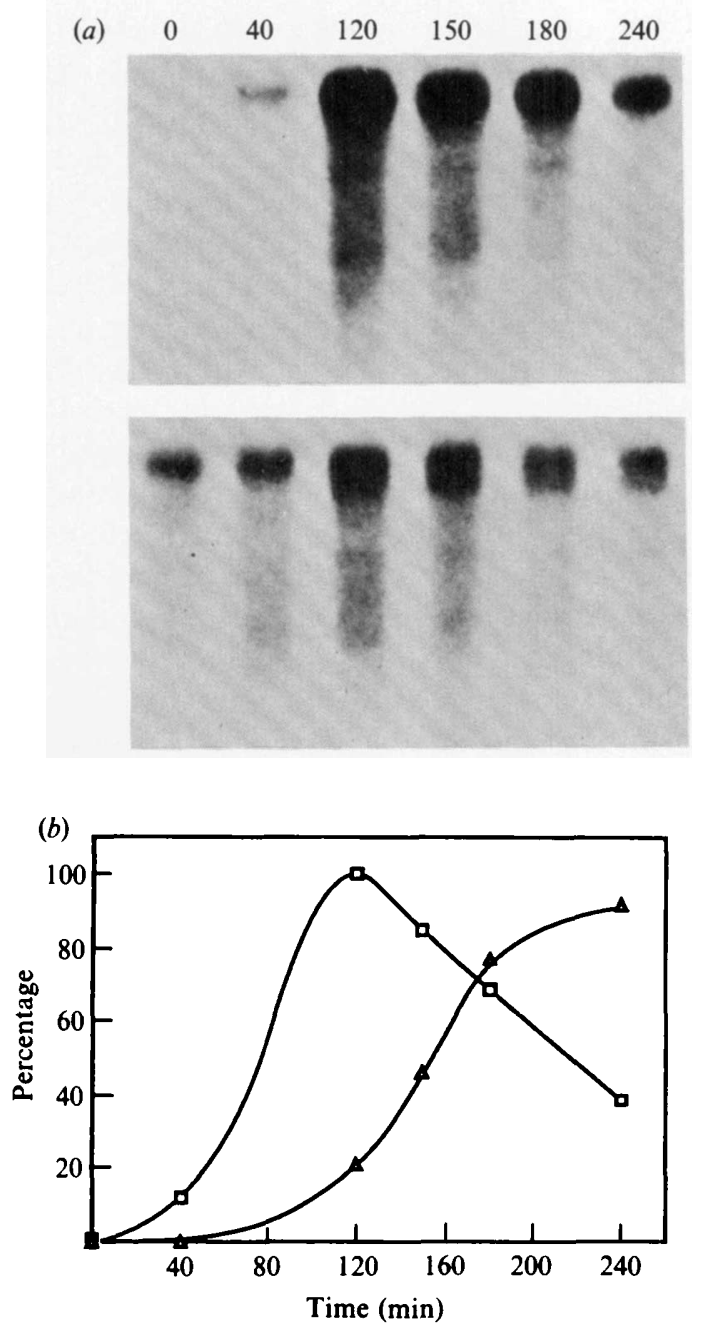

Fig. 6. (a) Northern blot analysis of total RNA isolated at different times after amoebae were induced to form flagellates. The numbers above each lane indicate the time in minutes after induction of flagellate development. Upper panel, probed with $\beta 501-33-3$, specific for bet $A$; lower panel, probed with $\beta 502$, which reacts with both bet $A$ and bet $B$ transcripts. The low level of bet $A$ transcript present in amoebae $(0 \mathrm{~min})$ is evident only on longer autoradiographic exposures. (b) Quantification of RNA ( $\square$, percentage of maximum) and percentage of flagellates $(\triangle)$ after induction of development.

normalized to $\mathrm{OD}_{260}$ units of RNA loaded for each sample.

\section{Discussion}

\section{Comparisons of Physarum $\beta$-tubulins}

One amino acid difference between the two Physarum $\beta 1$-tubulins had already been identified at position 283 by sequencing protein purified from amoebae (Singhofer-Wowra et al., 1986). In view of our observations of only a low level of betA transcript in amoebae, it is remarkable that readily detectable amounts of both alanine and serine could be found at position 283 by protein sequencing (Singhofer-Wowra et al., 1986). We suspect that manipulations used to harvest amoebae from culture induce elements of flagellate development, leading to increased levels of bet $A$ gene product in cell extracts.

Our cDNA sequencing indicated four other differences among Physarum $\beta 1$-tubulins, at residues 39,165 , 196 and 238 (Fig. 3). These differences might be expected simply to reflect differences between $\beta 1 \mathrm{~A}$ and $\beta 1 \mathrm{~B}$ tubulins, but only the differences at residues 39 and 238 seem to fit this interpretation. Each of these differences could have arisen by a change in a single nucleotide between bet $A$ and bet $B$. At position 165 , however, cysteine is indicated in both $\beta 602$ (bet $A$ ) and $\mathrm{p} \beta 40-21$ (bet $B$ ), but aspartate was found in the $\beta 1$ protein sequence (Singhofer-Wowra et al., 1986). Similarly, position 196 is alanine in $\beta 602$ and $\mathrm{p} \beta 40-21$, but threonine in the $\beta 1$ protein sequence. The Ala-Thr discrepancy at codon 196 could be accounted for by a single nucleotide change, whereas the Cys-Asp discrepancy would need two nucleotide changes. These discrepancies in the protein sequence where bet $A$ and bet $B$ DNA sequences appear isocoding could be due to errors in sequencing, strain differences, or post-transcriptional modifications. We discount the possibility of a third $\beta 1$-tubulin gene as this would surely have been detected by Southern blotting. The complete $\beta 1 \mathrm{~A}$ polypeptide remains $88-90 \%$ identical to $\beta$-tubulins from a broad range of organisms, including Chlamydomonas, Drosophila and vertebrates, but only $83 \%$ identical to Physarum $\beta 2$ tubulin, which is expressed primarily in plasmodia (Burland et al., 1988).

\section{Differential expression of $\beta$-tubulins}

During the transition of an amoeba into a flagellate, the level of bet $A$ transcript increases over 100-fold, while the level of the bet $B$ transcript changes very little. Based on the levels of bet $A$ and bet $B$ transcripts, we would expect that in amoebae, $\beta 1 \mathrm{~A}$-tubulin is of minor abundance and $\beta 1 \mathrm{~B}$-tubulin is the major $\beta$-tubulin; immunoblotting of axenic amoebal lysates supports this conclusion (Paul et al., 1989). In cultures radiolabelled as amoebae and induced to undergo the amoeba-flagellate transition, the $\beta 1 \mathrm{~A}$ polypeptide is radiolabelled more heavily than $\beta 1 \mathrm{~B}$ (Burland et al., 1984), indicating that the level of $\beta 1 \mathrm{~A}$ tubulin increases with the increase in its mRNA level. Some synthesis of $\beta 1 \mathrm{~B}$ tubulin continues, however, so both polypeptides may be abundant in flagellates. Since most cells seem not to have a mechanism for excluding expressed tubulins from MTs (e.g. Lewis et al., 1987), we would expect that both $\beta 1 \mathrm{~A}$-and $\beta 1 \mathrm{~B}$-tubulin are assembled into the flagellum. 
The prediction that $\beta 1 \mathrm{~A}-$ tubulin is of minor abundance in amoebae would contradict an earlier proposal that the two $\beta 1$-tubulins are of similar abundance in this cell type (Singhofer-Wowra et al., 1986), but would explain why mutation of bet $B$ alone is sufficient to confer resistance to antitubulin benzimidazoles on amoebae (Burland et al., 1984). However, if $\beta 1 \mathrm{~B}$-tubulin is indeed the principal $\beta$-tubulin of the amoeba, our earlier hypothesis that mutation of bet $A$ alone can also lead to benzimidazole resistance (Burland et al., 1984) would warrant further investigation.

Strong differential expression of Physarum $\beta$-tubulins is further illustrated during the developmental transition of an amoeba into a plasmodium. At some point during this transition betA transcripts disappear, and can no longer be detected in the plasmodium, while betC transcripts (encoding $\beta 2$-tubulin), which are not detected in the amoeba, are first diagnosed when cells become committed to plasmodium development (Solnica-Krezel et al., 1988). Our results show that the bet $B$ gene does not undergo such dramatic regulation as is seen for the bet $A$ and bet $C$ tubulin genes during these developmental transitions. However, in plasmodia, bet $B$ transcript and $\beta 1 \mathrm{~B}$-tubulin levels are significantly lower than betC transcript and $\beta 2$-tubulin levels (Burland et al., 1983; Solnica-Krezel et al., 1988). Thus, each of the three cell types we have studied preferentially expresses one of the three $\beta$-tubulin genes: bet $A$ in the flagellate, bet $B$ in the amoeba, and betC in the plasmodium.

\section{Regulation of $\beta$-tubulin expression}

Several examples of a rise in tubulin RNA levels during flagellar development have been noted. In Physarum, $\alpha-$ tubulin transcript levels increase five- to sevenfold over the same time frame that bet $A$ rises 110-fold (Green \& Dove, 1984). In Naegleria (Lai et al., 1988; Lee \& Walsh, 1988), $\alpha$-tubulin RNA is first detected $15 \mathrm{~min}$ after flagellate development is induced, and during flagellate development the level of $\alpha$-tubulin RNA increases dramatically. Similar increases in tubulin transcript levels during flagellar development have been extensively documented for Chlamydomonas (Lefebvre \& Rosenbaum, 1986), where increases in both transcription and stability of tubulin RNA have been noted (Baker et al., 1984). In contrast to Physarum, it appears that expression of the two isocoding $\beta$-tubulin genes of Chlamydomonas (Youngbloom et al., 1984), like that of the two $\alpha$-tubulin genes, is coordinately regulated during flagellar growth (Brunke et al., 1982). However, in the case of Chlamydomonas, the appearance of flagella occurs at a specific cell cycle stage, and is not associated with a change in cell type.
How do cells adjust mRNA levels when major physiological changes occur? For $\beta$-tubulin, there is evidence for negative feedback regulation of mRNA level by $\beta$-tubulin protein, in organisms as diverse as cultured mammalian cells (Yen et al., 1988) and sea urchin (Gong \& Brandhorst, 1987; Harlow \& Nemer, 1987); high levels of free tubulin lead, directly or indirectly, to decreased tubulin RNA levels. In mammalian cells, the first four codons are necessary for this negative regulation of $\beta$-tubulin transcript levels (Yen et al., 1988), and it is remarkable that the sequence of the first four amino acid residues is very highly conserved, including for all three Physarum $\beta$-tubulins (Burland $e t$ $a l .$, 1988). This mechanism could thus be important for tubulin expression in many organisms. Indeed, the timing of changes in tubulin transcript level with respect to flagellar assembly fits well with a negative regulatory mechanism. In flagellates of Physarum, Chlamydomonas and Naegleria, the peak level of tubulin transcripts occurs prior to the completion of flagellar development (Baker et al., 1984; Lai et al., 1988; Lee \& Walsh, 1988). By the time construction of the flagellar axoneme is completed, the level of tubulin RNA is on the decline. If these cells synthesize tubulin protein beyond the level required for the construction of axonemes, the increase in the level of free tubulin subunits could then homeostatically depress tubulin transcript level. It remains to be determined what mechanisms might turn on tubulin expression in advance of flagellar assembly.

\section{Functions of $\beta$-tubulins}

The bet $A$ and bet $B$ genes of Physarum have drifted from one another in their nucleotide sequence but their products nevertheless remain virtually identical in amino acid sequence. This implies that the duplication that gave rise to these two genes is ancient, but that there is a strong constraint on the $\beta 1$ tubulin polypeptides acting to conserve their sequences. The close similarity of the bet $A$ and bet $B$ gene products does not preclude a functional distinction between these two molecules, but we have no evidence to support such a distinction with respect to microtubular function.

Some evidence for preferential assembly of specific $\beta$ tubulins into MTs has been obtained, for example in differentiating neurites (Joshi \& Cleveland, 1989). However, most investigations have examined only participation in MTs by distinct $\beta$-tubulin isotypes of minor abundance, rather than active function by isotypes of major abundance. We previously noted that isotypes of minor abundance might be carried as 'passengers' in MTs, and predicted that genetic drift would occur to the extent that such passengers did not 
poison microtubular function (Burland et al., 1988). The demonstration that the Drosophila minor divergent $\beta 3$ tubulin isotype impairs functional axoneme assembly when it exceeds $20 \%$ of cellular $\beta$-tubulin provides experimental confirmation of this idea (Hoyle \& Raff, 1990). We would not expect to observe this particular distinction for Physarum $\beta 1 \mathrm{~A}$ and $\beta 1 \mathrm{~B}$ tubulins, since both are probably abundant during flagellate development. But perhaps high levels of expression of Physarum $\beta 2$-tubulin would impair the function of flagella.

Even where substantial polypeptide sequence differences are observed between multiple $\beta$-tubulins within a single cell, it has been difficult to find compelling evidence for distinct functional roles (e.g. Sullivan, 1988). For example, the divergent $\beta 2$ tubulin (the product of the betC gene) of Physarum has been detected in all the MT types observed during the amoebalplasmodial developmental transition, including cytoplasmic, open and closed mitotic-spindle, flagellar, and centriolar MTs (Diggins-Gilicinski et al., 1989; SolnicaKrezel et al., 1990).

The $17 \%$ difference in amino acid sequence between $\beta 1$ and $\beta 2$ tubulins is remarkable in view of the similarity between the $\beta 1 \mathrm{~A}$ and $\beta 1 \mathrm{~B}$ tubulins. Singhofer-Wowra $e t$ al. (1986) predicted that Physarum $\beta 2$-tubulin would be more diverged than $\beta 1$ tubulins since it usually participates only in mitosis and meiosis; they suggested that participation of the $\beta 1$ tubulins not only in the mitotic spindle but also in more complex organelles like the flagellum may constrain sequence divergence. This interpretation is consistent with the idea that both $\beta 1 \mathrm{~A}$ and $\beta 1 B$-tubulins are utilized in the flagellum, and would argue against the maintenance of the two $\beta 1$-tubulin genes for distinct microtubular functions. Our results indicate that the existence of the bet $A-$ bet $B$ gene pair provides a mechanism for strong differential regulation of tubulin genes in different cell types, and the selective advantage of this mechanism may be the evolutionary force that maintains both genes.

We thank Laurie Larson for excellent technical assistance, Julie Ahringer for advice on constructing the cDNA library, Valerie Burland for advice on preparing packaging extracts, and our colleagues for helpful comments on the manuscript.

This research was supported by program project grant CA23076 and by core grant CA07175 from the National Cancer Institute. E. C.A.P. was supported by postdoctoral training grant 2-T32-CA09230 from the National Cancer Institute.

\section{References}

Aviv, H. \& LeDER, P. (1972). Purification of biologically active globin messenger RNA by chromatography on oligothymidylic acid cellulose. Proceedings of the National Academy of Sciences of the United States of America 69, 1408-1418.
Baker, E. J., Schloss, J. A. \& Rosenbaum, J. L. (1984). Rapid changes in tubulin RNA synthesis and stability induced by deflagellation in Chlamydomonas. Journal of Cell Biology 99, 2074-2081.

BirKetT, C. R., Foster, K. E., Johnson, L. \& GulL, K. (1985). Use of monoclonal antibodies to analyse the expression of a multitubulin gene family. FEBS Letters 187, 211-218.

Brunke, K. J., Young, E. E., Buchinder, B. U. \& Weeks, D. P. (1982). Coordinate regulation of the four tubulin genes of Chlamydomonas reinhardi. Nucleic Acids Research 10, 1295-1310.

Burland, T. G., Gull, K., Schedl, T., Boston, R. S. \& Dove, W. F. (1983). Cell-type dependent expression of tubulins in Physarum. Journal of Cell Biology 97, 1852-1859.

Burland, T. G., SChedL, T., Gull, K. \& Dove, W. F. (1984). Genetic analysis of resistance to benzimidazoles in Physarum: differential expression of beta tubulin genes. Genetics 108, 123-141.

Burland, T. G., Paul, E. C. A., Oetliker, M. \& Dove, W. F. (1988). A gene encoding the major beta-tubulin of the mitotic spindle in Physarum polycephalum plasmodia. Molecular and Cellular Biology 8 , 1275-1281.

COOKE, D. J. \& DEE, J. (1975). Methods for the isolation and analysis of plasmodial mutants in Physarum polycephalum. Genetical Research 24, 175-187.

Dale, R. M. K., McClure, B. A. \& Houchins, J. P. (1985). A rapid single-stranded cloning strategy for producing a sequential series of overlapping clones for use in DNA sequencing: application to sequencing the corn mitochondrial 18S rDNA. Plasmid 13, 31-40.

DeE, J., Foxon, J. L. \& ANDERson, R. W. (1989). Growth, development and genetic characteristics of Physarum polycephalum amoebae able to grow in liquid, axenic medium. Journal of General Microbiology 135, 1567-1588.

Diggins-Gilicinski, M., Solnica-Krezel, L., Burland, T. G., Paul, E. C. A. \& Dove, W. F. (1989). The localization of the divergent $\beta 2-$ tubulin in the microtubular arrays of Physarum polycephalum. Journal of Cell Science 94, 217-226.

FuLTON, C. \& Simpson, P. A. (1976). Selective synthesis and utilization of flagellar tubulin : the multi-tubulin hypothesis. In Cell Motility, pp. 987-1005. Edited by R. Goldman, T. Pollard \& J. Rosenbaum. Cold Spring Harbor, NY: Cold Spring Harbor Laboratory.

GoNG, Z. \& BRANDHORST, B. P. (1987). Stimulation of tubulin gene transcription by deciliation of sea urchin embryos. Molecular and Cellular Biology 7, 4238-4246.

Green, L. \& Dove, W. F. (1984). Tubulin proteins and RNA during the myxamoeba-flagellate transformation of Physarum polycephalum. Molecular and Cellular Biology 4, 1706-1711.

Harlow, P. \& Nemer, M. (1987). Developmental and tissue-specific regulation of $\beta$-tubulin gene expression in the embryo of the sea urchin Strongylocentrotus purpuratus. Genes and Development 1, 147160.

Hoyle, H. D. \& RAFf, E. C. (1990). Two Drosophila bet tubulin isoforms are not functionally equivalent. Journal of Cell L.ology 111, 1009-1026.

HuYNH, T. V., Young, R. A. \& DAvis, R. W. (1984). Constructing and screening cDNA libraries in lambda gt 10 and lambda gt 11 . In DNA Cloning Techniques: A Practical Approach, pp. 49-78. Edited by D. Glover. Oxford: IRL Press.

Joshi, H. C \& CLEveland, D. W. (1989). Differential utilization of $\beta$ tubulin isotypes in differentiating neurites. Journal of Cell Biology 109, 663-675.

Kilmartin, J. V., Wright, B. \& Milstein, C. (1982). Rat monoclonal antitubulin antibodies derived using a new non-secreting rat cell line. Journal of Cell Biology 93, 576-582.

LAI, E.Y, Remillard, S. P. \& Fulton, C. (1988). The $\alpha$-tubulin gene family expressed during cell differentiation in Naegleria gruberi. Journal of Cell Biology 106, 2035-2046.

LASKEY, R. A. \& MiLLS, A. D. (1977). Enhanced autoradiographic detection of ${ }^{32} \mathrm{P}$ and ${ }^{125} \mathrm{I}$ using intensifying screens and hypersensitized films. FEBS Letters 82, 314-322.

LEE, J. H. \& WALSH, C. J. (1988). Transcriptional regulation of coordinate changes in flagellar mRNAs during differentiation of Naegleria gruberi amebae into flagellates. Molecular and Cellular Biology 8, 2280-2287. 
Lefebvre, P. A. \& Rosenbaum, J. L. (1986). Regulation of the synthesis and assembly of ciliary and flagellar proteins during regeneration. Annual Review of Cell Biology 2, 517-546.

LEWIS, S. A., Gu, W. \& CowaN, N. J. (1987). Free intermingling of mammalian $\beta$-tubulin isotypes among functionally distinct microtubules. Cell 49, 539-548.

Melton, D. A., Kreig, P. A., Rebagliati, M. R., Maniatis, T., ZinN, K. \& GREeN, M. R. (1984). Efficient in vitro synthesis of biologically active RNA and RNA hybridization probes from plasmids containing a bacteriophage SP6 promoter. Nucleic Acids Research 12, 7035-7070.

MERCER, J. F. B. \& WAKE, S. A. (1985). An analysis of the rate of metallothionein mRNA poly(A) shortening using RNA blot hybridization. Nucleic Acids Research 13, 7929-7943.

NeEdleman, S. B. \& WunsCh, C. D. (1970). A general method applicable to the search for similarities in the amino acid sequence of two proteins. Journal of Molecular Biology 48, 443-453.

Paul, E. C. A., Burland, T. G. \& Gull, K. (1989). Location of a single $\beta$-tubulin gene product in both cytoskeletal and mitoticspindle microtubules in Physarum polycephalum. Journal of General Microbiology 135, 623-628.

RAFF, E. C. (1984). Genetics of microtubule systems. Journal of Cell Biology 99, 1-10.

Rigby, P. W., Dieckman, M., Rhodes, C. \& Berg, P. (1977). Labelling deoxyribonucleic acid to high specific activity in vitro by nick translation with DNA polymerase I. Journal of Molecular Biology 113, 237-332.

Sanchez, F., Natzle, J. E., Cleveland, D. W., Kirschner, M. W. \& MCCARTHY, B. J. (1980). A dispersed multigene family encoding tubulin in Drosophila melanogaster Cell 22, 845-854.

SANGer, F., Nicklen, S. \& Coulson, A. R. (1977). DNA sequencing with chain terminating inhibitors. Proceedings of the National Academy of Sciences of the United States of America 74, 5463-5467.

Schedl, T., Burland, T. G., Gull, K. \& Dove, W. F. (1984a). Cell cycle regulation of tubulin RNA level, tubulin protein synthesis, and assembly of microtubules in Physarum. Journal of Cell Biology 99. 155-165.

SChedl, T., Owens, J., Dove, W. F. \& Burland, T. G. (1984b) Genetics of the tubulin gene families of Physarum. Genetics 108, 143164.
Singhofer-Wowra, M., Clayton, L., Dawson, P., Gull, K. \& Litrle, M. (1986). Amino-acid sequence data of $\beta$-tubulin from Physarum polycephalum myxamoebae. European Journal of Biochemistry 161, 669-679.

Solnica-Krezel, L., Dove, W. F. \& Burland, T. G. (1988). Activation of a $\beta$-tubulin gene during early development of the plasmodium in Physarum polycephalum. Journal of General Microbiology 134, 1323-1331.

Solnica-Krezel, L., Diggins-Gilicinski, M., Burland, T. G. \& Dove, W. F. (1990). Variable pathways for developmental changes in composition and organization of microtubules in Physarum polycephalum. Journal of Cell Science 96, 383-393.

SOUTHERN, E. M. (1975). Detection of specific sequences among DNA fragments separated by gel electrophoresis. Journal of Molecular Biology 98, 503-517.

Sullivan, K. F. (1988). Structure and utilization of tubulin isotypes. Annual Reviews of Cell Biology 4, 687-716.

VAlenzuela, P., QUiRoga, M., Zaldivar, J., RUTter, W. J., Kirschner, M. W. \& Cleveland, D. W. (1979). Nucleotide and corresponding amino acid sequences encoded by $\alpha$ and $\beta$ tubulin mRNAs. Nature, London 289, 650-655.

Walden, E. D., Blindt, A. B., Birkett, C. R., Cox, R. A. \& Gull, K. (1989). Recognition of specific Physarum $\alpha$-tubulin isotypes by a monoclonal antibody. European Journal of Biochemistry 185, 383389.

Werenskiold, A. K., Poetsch, B. \& Haugli, F. (1988). Cloning and expression of a $\beta$-tubulin gene of Physarum polycephalum. FEBS Letters 243, 491-495.

Yen, T. J., Gay, D. A., Pachter, J. S. \& Cleveland, D. W. (1988). Autoregulated changes in stability of polyribosome-bound $\beta$-tubulin mRNAs are specified by the first 13 translated nucleotides. Molecular and Cellular Biology 8, 1224-1235.

Youngbloom, J., Schloss, J. A. \& Silflow, C. D. (1984). The two $\beta$ tubulin genes of Chlamydomonas reinhardtii code for identical proteins. Molecular and Cellular Biology 4, 2686-2696.

ZehNBaUer, B. A. \& Blattrer, F. R. (1982). Construction and screening of recombinant DNA libraries with Charon vector phages. In Genetic Engineering vol. 4, pp. 249-279. Edited by J. K. Setlow \& A. Hollaender. New York: Plenum. 